\title{
EXTENSION METHODS IN CARDINAL ARITHMETIC
}

\author{
BY \\ ERIK ELLENTUCK( $\left.{ }^{1}\right)$
}

\begin{abstract}
Functions (relations) defined on the nonnegative integers are extended to the cardinal numbers by the method of Myhill (Nerode) respectively. We obtain various results relating these extensions and conclude with an analysis of AE Horn sentences interpreted in the cardinal numbers. Let $\mathfrak{A}$ be the sentence $\left(\forall x_{1}\right) \ldots$ $\left(\forall x_{n}\right)(\exists ! y) \mathfrak{b}$ where quantifiers are restricted to the Dedekind cardinals and $\mathfrak{b}$ is an equation built up from functors for cardinal addition, multiplication, and integer constants. One of our principal results is that $\mathfrak{A}$ is a theorem of set theory (with the axiom of choice replaced by the axiom of choice for sets of finite sets) if and only if we can prove that the uniquely determined Skolem function for $\mathfrak{A}$ extends an almost combinatorial function.
\end{abstract}

1. Introduction. Let $\omega=$ the nonnegative integers, $\Lambda=$ the isols (cf. [3]), and $\Delta=$ the Dedekind cardinals. For $0<k<\omega$ use $\times{ }^{k} A$ for the $k$-fold direct power of $A$. Recursive combinatorial functions were introduced by Myhill (cf. [11]) in order to study cancellation laws in isolic arithmetic. His method consists in first showing that each recursive combinatorial $f: \times{ }^{k} \omega \rightarrow \omega$ can be extended to an $f_{\Lambda}: \times{ }^{k} \Lambda \rightarrow \Lambda$. He then proves that a cancellation law, involving recursive combinatorial $f$ 's, and holding in $\omega$, implies a corresponding cancellation law, involving the $f_{\Lambda}$ 's, and holding in $\Lambda$. Results of this type are generally referred to as transfer theorems (from $\omega$ to $\Lambda$, in this case). Later, Nerode gave a method (cf. [12]) whereby a recursive $R \subseteq \times{ }^{k} \omega$ could be extended to an $R_{\Lambda} \subseteq \times^{k} \Lambda$, and found the exact relationship between his extension and Myhill's. Further, he went on to generalize Myhill's results on cancellation laws, and found the transfer theorem, from $\omega$ to $\Lambda$, for first order universal sentences involving recursive $R$ 's and recursive combinatorial $f$ 's. As a byproduct of this analysis, Nerode introduced a number of concepts closely related to the combinatorial functions; namely, eventually combinatorial and almost combinatorial functions. He then showed that the class of recursive almost combinatorial functions is in a certain sense (cf. Metacorollary 1 of this paper) maximal with respect to extension methods of either the Myhill or Nerode type.

Received by the editors July 30, 1969.

AMS Subject Classifications. Primary 0260.

Key Words and Phrases. Dedekind cardinal, universal cardinal, combinatorial function, eventually combinatorial function, almost combinatorial function, combinatorial operator, frame, Horn sentence.

(1) We take this opportunity to thank Professor J. C. E. Dekker for his continuing encouragement regarding our area of research. This paper was prepared while the author was supported by a New Jersey Research Council Faculty Fellowship.

Copyright (C) 1970, American Mathematical Society 
Combinatorial methods have proved to be equally useful for the study of Dedekind cardinals without the axiom of choice. In [5] combinatorial functions were extended from $\omega$ to $\Delta$ and a transfer theorem, similar to Nerode's, was obtained from $\omega$ to $\Delta$, for first order universal sentences involving combinatorial $f$ 's. Relations, eventually combinatorial, and almost combinatorial functions were not discussed as they appeared to be unnecessary from an algebraic viewpoint. However in a later work (cf. [8]) we found that almost combinatorial functions play a crucial role in the many quantifier theory of $\Delta$. For that reason we devote this paper to a general exploration of extension methods in cardinal arithmetic.

In $\$ 2$ we use a modification of Nerode's extension method (cf. [12]) to extend an $R \subseteq \times^{k} \omega$ to an $R_{\Delta} \subseteq \times^{k} \Delta$, and relate this to the extension of combinatorial functions (cf. [5]). In $\S 3$ we discuss eventually combinatorial and almost combinatorial functions, and in $\$ 4$ we show that the class of almost combinatorial functions is maximal with respect to our extension methods. In $\$ 5$ we compute cardinalities of the various Boolean combinations of the $R_{\Delta}$ 's, and in $\S 6$ the fundamental result of [14] (cf. (37)) is used in order to give an analysis of extending Horn sentences. Throughout this paper we will try to avoid favoring either a functional or a relational approach to $\Delta$ (thus 'we omit a discussion of frame maps), but rather we will show how a complementary study of both extension methods leads to a smooth analysis of $\Delta$.

The following discussion takes place within the set theory $\mathbb{B S}^{\circ}$ consisting of axioms $\mathrm{A}-\mathrm{D}$ of $[9]+\mathrm{AC}^{0}$ (=the axiom of choice for sets of finite sets). Denote ordinals (finite ordinals) by lower case Greek (Latin) letters, and cardinals (finite cardinals) by lower case German (Latin) letters. Finite ordinals and cardinals are identified in the usual way under the joint heading of integers. Use $|A|$ for the cardinal of the set $A$, and let $\Gamma$ be class of all cardinals, $\omega=$ the set of all integers, and $\omega^{*}=$ the full ring of rational integers. Let $\aleph_{0}=|\omega|$, call a Dedekind if $\aleph_{0}$ a , and let $\Delta=$ the class of all Dedekind cardinals, $\Delta^{\infty}=\Delta-\omega, \Delta^{*}=$ the full ring of Dedekind integers (cf. [6]). A set is finite, Dedekind, Dedekind infinite, countable, or countably infinite if its cardinal $\in \omega, \in \Delta, \in \Delta^{\infty}, \leqq \boldsymbol{\aleph}_{0}$, or $=\boldsymbol{\aleph}_{0}$ respectively. Let $\boldsymbol{V}$ be the class of all sets, $\boldsymbol{D}=$ the class of all Dedekind sets, and $\boldsymbol{Q}=$ the class of all finite sets. Use $A \cup(\cap) B$ for the union (intersection) of $A$ and $B, \cup(\bigcap) A$ for the union (intersection) of the members of $A, \mathfrak{P}(A)=$ the class of all subsets of $A$ and $\mathfrak{P}_{\omega}(A)=\mathfrak{P}(A) \cap \boldsymbol{Q}$. If $F$ is a function write $y=F(x)$ for $\langle y, x\rangle \in F$ and let $\delta F(\rho F)$ stand for domain (range) of $F$. Let $F \mid A=\{\langle y, x\rangle \in F: x \in A\}$. Write $F: A \rightarrow B$ for $A \subseteq \delta F$ and $\rho(F\lceil A) \subseteq B$. Write $F: A \sim B$ for $F$ is one one, $A \subseteq \delta F$ and $\rho(F\lceil A)=B$. If in the latter case we are indifferent to $F$, simply write $A \sim B$. We denote the composition of functions $F$ and $G$ by $F \circ G$ and associate in such a way that $(F \circ G)(x)=F(G(x))$. For any class $A$ and $k \in \omega$ let $\times^{k} A$ be the class of all functions $x: k \rightarrow A$ having $\delta x=k$. Members of $\times^{k} V$ are called $k$-tuples and are exhibited as $x=\left\langle x_{0}, \ldots, x_{k-1}\right\rangle$ where we write $x_{i}$ for $x(i)$. Let $\wedge$ be the binary concatenation operation on tuples. A function $F$ with $\delta F \subseteq \times^{k} V$ is called a $k$-ary 
function. In this case write $F\left(x_{0}, \ldots, x_{k-1}\right)$ in place of $F\left(\left\langle x_{0}, \ldots, x_{k-1}\right\rangle\right)$ and $\left(F \circ\left(G_{0}, \ldots, G_{k-1}\right)\right)(x)=F\left(G_{0}(x), \ldots, G_{k-1}(x)\right)$ for $k$-ary composition. Many of our notions initially defined on $\boldsymbol{V}$ are extended componentwise from $\boldsymbol{V}$ to $X^{k} \boldsymbol{V}$. This will usually be denoted by appending a "*" as a superscript to the symbol for the unextended notion. Thus if $A, B \in X^{k} V, R$ is a binary relation, and $F$ is a unary function, we write $R^{*}(A, B), F^{*}(A)=B$ whenever $R\left(A_{i}, B_{i}\right), F\left(A_{i}\right)=B_{i}$ for each $i<k$. This notation does not always work smoothly, particularly when we negate a concept, so that in many cases we will have to spell out the exact definition of the extended notion.

2. Extending relations. In the following discussion let $0<k<\omega$ be fixed. A $k$-frame (modified [12]) is a set $F \subseteq X^{k} \boldsymbol{Q}$ which satisfies

(1) if $A, A^{\prime} \in F$ then $A \cap^{*} A^{\prime} \in F$.

(2) Let $\mathscr{A}(F)=\left\{B \in X^{k} V:\left(\forall A \in X^{k} Q\right)\left(\exists A^{\prime} \in F\right)\left(A \subseteq{ }^{*} B \rightarrow A \subseteq{ }^{*} A^{\prime} \subseteq{ }^{*} B\right)\right\}$.

Elements in $\mathscr{A}(F)$ are said to be attainable from $F$.

(3) Let $F^{e}=\left\{A \in X^{k} Q:(\exists B)\left(A \subseteq{ }^{*} B \in F\right)\right\}$

(4) and $\gamma_{F}(A)=\bigcap^{*}\left\{B \in \times^{k} Q: A \subseteq * B \in F\right\}$.

It is easy to see that if $F$ is a frame and $A \in F^{e}$ then $\gamma_{F}(A)$ is the minimal element $B$, with respect to $\subseteq^{*}$, which satisfies $A \subseteq{ }^{*} B \in F$.

Let $R \subseteq X^{k} \omega$ be a relation. A $k$-frame $F$ is called an $R$-frame if $|A|^{*} \in R$ for every $A \in F$. Then we have our main Definition 1 (modified [12]): For $R \subseteq \times^{k} \omega$ let

$$
R_{\Gamma}=\left\{\mathfrak{a} \in X^{k} \Gamma:\left(\exists A \in X^{k} V\right)(\exists \text { an } R \text {-frame } F)\left(\mathfrak{a}=|A|^{*} \text { and } A \in \mathscr{A}(F)\right)\right\} .
$$

If $\Sigma \subseteq \Gamma$ let $R_{\Sigma}=R_{\Gamma} \cap \times^{k} \Sigma$. Write $R_{\Sigma}(\mathfrak{a})$ to mean $\mathfrak{a} \in R_{\Sigma}$.

Note that frames have been defined as sets in order to avoid needing the $\Sigma_{1}^{1}$ comprehension axiom to guarantee the existence of $R_{\Gamma}$. Since $\mathscr{A}(F) \cap \times^{k} \boldsymbol{Q}=F$ for any frame $F$, we have

(5) if $R \subseteq \times^{k} \omega$ then $R_{\omega}=R$.

Let $f: X^{k} \omega \rightarrow \omega$ be a combinatorial function and let $f_{\Gamma}$ be its canonical extension to $\Gamma$. If $\Sigma \subseteq \Gamma$ let $f_{\Sigma}=f_{\Gamma} \uparrow \times^{k} \Sigma$. It follows from [5] that

(6) if $f$ is a combinatorial function then $f_{\omega}=f$.

In [4] we gave an entirely different definition of $R_{\Delta}$. In order to justify that definition it will be useful to have the following facts on hand. If $R \subseteq X^{k} \omega$ then

$$
R=\left\{a \in X^{k} \omega: f_{0}(a)=f_{1}(a)\right\}
$$

for some pair of combinatorial functions $f_{0}, f_{1}$. This follows from the fact that every function $f: X^{k} \omega \rightarrow \omega$ can be expressed as the difference of two combinatorial functions. A fortiori so can the characteristic function of $R$.

Let $L$ be a language with identity containing variables and functors. If $\mathfrak{A}$ is a sentence of $L$ and $\Sigma \subseteq \Gamma$ let $\mathfrak{A}_{\Sigma}$ be the interpretation obtained by allowing variables to range over $\Sigma$ and letting functors denote extensions to $\Sigma$ of fixed combinatorial 
functions. If $\mathfrak{A}$ is a universally quantified prenex normal form Horn sentence then

(8) if $\mathfrak{A}_{\omega}$ then $\mathfrak{A}_{\Delta}$ (which is Theorem 8 of [5]).

These results justify our second important

Definition 1' (of [4]). For $R \subseteq \times^{k} \omega$ let

$$
R^{\Delta}=\left\{\mathfrak{a} \in X^{k} \Delta: f_{0, \Delta}(\mathfrak{a})=f_{1, \Delta}(\mathfrak{a})\right\}
$$

where $f_{0}, f_{1}$ are any pair of combinatorial functions satisfying (7). If $\Sigma \subseteq \Delta$ let let $R^{\Sigma}=R^{\Delta} \cap \times^{k} \Sigma$. Write $R^{\Sigma}(\mathfrak{a})$ to mean $\mathfrak{a} \in R^{\Sigma}$.

Note that (8) is used to show that $R^{\Delta}$ is independent of the particular $f_{0}, f_{1}$ satisfying (7). By (6) it is immediate that

(9) if $R \subseteq \times^{k} \omega$ then $R^{\omega}=R$.

The principal result of this section is $R_{\Delta}=R^{\Delta}$. We need

LEMMA 1. If $\Phi_{0}, \Phi_{1}$ are a pair of $k$-ary combinatorial operators, $B \in X^{k} D$, and $h: \Phi_{0}(B) \sim \Phi_{1}(B)$ then for every $A \in \times^{k} Q$, with $A \subseteq{ }^{*} B$ there is an $A^{\prime} \in X^{k} Q$ with $A \subseteq{ }^{*} A^{\prime} \subseteq{ }^{*} B$ such that $h: \Phi_{0}\left(A^{\prime}\right) \sim \Phi_{1}\left(A^{\prime}\right)$. (This is Lemma 5 of [5].)

Proof. Suppose that for some $A^{0} \in \times^{k} Q$ with $A^{0} \subseteq * B$ our lemma fails. Define a sequence $\left\langle A^{n}: n<\omega\right\rangle$ satisfying $A^{n} \in X^{k} Q, A^{n} \subseteq^{*} A^{n+1} \subseteq * B, A^{n} \neq A^{n+1}$ for every $n \in \omega$. The definition is given by the following induction. By hypothesis non $h: \Phi_{0}\left(A^{n}\right) \sim \Phi_{1}\left(A^{n}\right)$. Hence either (i) there is an $x \in \Phi_{0}\left(A^{n}\right)$ such that $y=h(x) \notin \Phi_{1}\left(A^{n}\right)$, or (ii) there is a $y \in \Phi_{1}\left(A^{n}\right)$ such that $x=h^{-1}(y) \notin \Phi_{0}\left(A^{n}\right)$, or (iii) both. Use AC ${ }^{0}$ to: in case (i) choose such an $x$ and define $A^{n+1}=A^{n} \cup^{*} \Phi_{1}^{-1}(y)$, or if (i) does not occur then (ii) does, so choose such a $y$ and define $A^{n+1}=A^{n} \cup^{*} \Phi_{0}^{-1}(x)$. Clearly the sequence we define this way satisfies the required conditions. Let $B^{\prime}=$ $\bigcup^{*}\left\{A^{n}: n<\omega\right\}, B^{\prime} \subseteq{ }^{*} B$ and is infinite in at least one component, say the $j$ th. Then the collection $\left\{A_{j}^{n+1}-A_{j}^{n}: n<\omega\right\}$ consists of pairwise disjoint finite sets, infinitely many of which are nonempty. Use $\mathrm{AC}^{0}$ to choose a set $C$ containing exactly one element from each nonempty $A_{j}^{n+1}-A_{j}^{n}$, but $|C|=\boldsymbol{\aleph}_{0}$ which contradicts $C \subseteq B_{j}^{\prime} \subseteq B_{j} \in$ D. Q.E.D.

Let us define

$$
\Phi^{F}(B)=\Phi(B)-\bigcup\{\Phi(A): A \in F \wedge A \subseteq * B \wedge A \neq B\} .
$$

LEMMA 2. If $\Phi$ is a $k$-ary combinatorial operator and $F$ is a $k$-frame then

(i) $\Phi^{F}(A) \cap \Phi^{F}\left(A^{\prime}\right)=\varnothing$ for $A, A^{\prime} \in F$ with $A \neq A^{\prime}$, and

(ii) $\Phi(B)=\bigcup\left\{\Phi^{F}(A): A \in F \wedge A \subseteq{ }^{*} B\right\}$ for $B \in \mathscr{A}(F)$.

Proof of (i). If $x \in \Phi^{F}(A) \cap \Phi^{F}\left(A^{\prime}\right)$ then $x \in \Phi(A) \cap \Phi\left(A^{\prime}\right)=\Phi\left(A \cap \cap^{*} A^{\prime}\right)$ by (10) and Lemma 1(ii) of [5]. Now $A \cap^{*} A^{\prime} \in F$ by (1), and $A \cap^{*} A^{\prime} \subseteq \subseteq^{*} A, A^{\prime}$. Thus if $A \cap^{*} A^{\prime} \neq A$ then $x \notin \Phi^{F}(A)$ by (10) which contradicts our hypothesis. Similarly for $A^{\prime}$. Hence $A=A \cap^{*} A^{\prime}=A^{\prime}$. Q.E.D.

Proof of (ii). If $A \subseteq{ }^{*} B$ then $\Phi^{F}(A) \subseteq \Phi(A) \subseteq \Phi(B)$ by (10) and Lemma 1(i) of [5]. Thus $\bigcup\left\{\Phi^{F}(A): A \in F \wedge A \check{\complement}^{*} B\right\} \subseteq \Phi(B)$. Conversely if $x \in \Phi(B)$ then $\Phi^{-1}(x) \subseteq{ }^{*} B$. But $B \in \mathscr{A}(F)$ hence by (2) and (3) $\Phi^{-1}(x) \in F^{e}$. If $A=\gamma_{F}\left(\Phi^{-1}(x)\right)$ then $A \in F$ and 
satisfies $\Phi^{-1}(x) \subseteq^{*} A \subseteq{ }^{*} B$. Clearly $x \in \Phi(A)$. If $x \notin \Phi^{F}(A)$ then by (10) $x \in \Phi\left(A^{\prime}\right)$ for some $A^{\prime} \in F$ with $A^{\prime} \subseteq \subseteq^{*} A$ but $A^{\prime} \neq A$. Then $\Phi^{-1}(x) \subseteq{ }^{*} A^{\prime}$, giving $\gamma_{F}\left(\Phi^{-1}(x)\right)$ $\subseteq^{*} A^{\prime}$ by (4), i.e., $A^{\prime}=A$, a contradiction. Thus $x \in \Phi^{F}(A)$. Q.E.D.

THEOREM 1. If $R \subseteq \times^{k} \omega$ then $R_{\Delta}=R^{\Delta}$.

Proof of $R^{\Delta} \subseteq R_{\Delta}$. Let $\mathfrak{b} \in R^{\Delta}, B \in X^{k} D$ with $\mathfrak{b}=|B|^{*}, f_{0}, f_{1}$ a pair of combinatorial functions satisfying (7), and $\Phi_{0}, \Phi_{1}$ a pair of combinatorial operators inducing $f_{0}, f_{1}$ respectively. By Definition $1^{\prime} f_{0, \Delta}(\mathfrak{b})=f_{1, \Delta}(\mathfrak{b})$ and hence there is a function $h: \Phi_{0}(B) \sim \Phi_{1}(B)$. Let $F=\left\{A \in X^{k} Q: A \subseteq * B \wedge h: \Phi_{0}(A) \sim \Phi_{1}(A)\right\}$. Clearly $F$ is a set. If $A, A^{\prime} \in F$ then $h: \Phi_{0}(A) \cap \Phi_{0}\left(A^{\prime}\right) \sim \Phi_{1}(A) \cap \Phi_{1}\left(A^{\prime}\right)$. But $\Phi_{j}(A)$ $\cap \Phi_{j}\left(A^{\prime}\right)=\Phi_{j}\left(A \cap^{*} A^{\prime}\right)$ by Lemma 1(ii) of [5], and hence $A \cap^{*} A^{\prime} \in F$. Moreover if $A \in F$ and $a=|A|^{*}$ then $a \in R$ since $f_{0}(a)=f_{1}(a)$. Thus $F$ is an $R$-frame from which $B$ is attainable by Lemma 1 . Hence $\mathfrak{b} \in R_{\Delta}$. Q.E.D.

Proof of $R_{\Delta} \subseteq R^{\Delta}$. Let $\mathfrak{b} \in R_{\Delta}, B \in X^{k} D$ with $\mathfrak{b}=|B|^{*}$, and $F$ an $R$-frame from which $B$ is attainable. Let $f_{0}, f_{1}$ be a pair of combinatorial functions satisfying (7), and $\Phi_{0}, \Phi_{1}$ a pair of combinatorial operators inducing $f_{0}, f_{1}$ respectively. For $A \in F$ we show by induction on $|A|^{*}$ that $\Phi_{0}^{F}(A) \sim \Phi_{1}^{F}(A)$. Suppose the result is true for all $A^{\prime} \in F$ where $\left|A^{\prime}\right|^{*} \leqq *|A|^{*}$ but $\left|A^{\prime}\right|^{*} \neq|A|^{*}$. Then

$$
\Phi_{j}(A)=\Phi_{j}^{F}(A) \cup \bigcup\left\{\Phi_{j}^{F}\left(A^{\prime}\right): A^{\prime} \in F \wedge A^{\prime} \subseteq^{*} A \wedge A^{\prime} \neq A\right\}
$$

follows from Lemma 2(ii) since $A \in F \subseteq \mathscr{A}(F)$. Now $A \in F$ and $a=|A|^{*}$ implies $a \in R$, i.e., $f_{0}(a)=f_{1}(a)$ by (7) and consequently $\Phi_{0}(A) \sim \Phi_{1}(A)$. By induction hypothesis $\Phi_{0}^{F}\left(A^{\prime}\right) \sim \Phi_{1}^{F}\left(A^{\prime}\right)$ for $A^{\prime} \in F, A^{\prime} \subseteq{ }^{*} A$ with $A^{\prime} \neq A$. But the union in (11) is disjoint by Lemma 2(i) and hence $\Phi_{0}^{F}(A) \sim \Phi_{1}^{F}(A)$ by the uniqueness of subtraction for finite sets. Now $B \in \mathscr{A}(F)$, so that by Lemma 2(ii)

$$
\Phi_{j}(B)=\bigcup\left\{\Phi_{j}^{F}(A): A \in F \wedge A \subseteq \subseteq^{*} B\right\}
$$

where the union is disjoint. Use $\mathrm{AC}^{0}$ to choose maps $h_{A}: \Phi_{0}^{F}(A) \sim \Phi_{1}^{F}(A)$ for $A \in F$ with $A \subseteq{ }^{*} B$. Clearly by the above disjointness

$$
\bigcup\left\{h_{A}: A \in F \wedge A \subseteq \subseteq^{*} B\right\}=h: \Phi_{0}(B) \sim \Phi_{1}(B) .
$$

Thus $f_{0, \Delta}(\mathfrak{b})=f_{1, \Delta}(\mathfrak{b})$ and consequently $\mathfrak{b} \in R^{\Delta}$. Q.E.D.

Let LR be a language with identity containing variables, functors and predicates. If $\mathfrak{A}$ is a sentence of $L R$ and $\Sigma \subseteq \Gamma$ let $\mathfrak{A}_{\Sigma}$ be the interpretation obtained by allowing variables to range over $\Sigma$, by letting functors denote extensions to $\Sigma$ of fixed combinatorial functions, and by letting predicates denote extensions to $\Sigma$ (by Definition 1) of fixed relations over $\omega$. We generally assume that sentences are all written in prenex conjunctive normal form.

If $\mathfrak{A}$ is a universally quantified Horn sentence we have

THEOREM 2. If $\mathfrak{A}_{\omega}$ then $\mathfrak{A}_{\Delta}$.

Proof. Use Theorem 1 and the fact that if we extend relations by Definition 1' the result will follow by (8). Q.E.D. 
Indeed, the idea in Theorem 7 of [5], and the universal cardinals of [7], can be used to obtain a complete reduction to $\omega$, of the provability of $\mathfrak{A}_{\Delta}$, for universally quantified sentences $\mathfrak{A}$. However the preceding theorem will be sufficient for the purposes of this paper.

3. Extending functions. In Definition 2 of [6] we extended every function $f: \times^{k} \omega \rightarrow \omega^{*}$ to a function $f_{\Delta}: X^{k} \Delta \rightarrow \Delta^{*}$ by defining

$$
f_{\Delta}(\mathfrak{a})=f_{\Delta}^{+}(a)-f_{\Delta}^{-}(\mathfrak{a}) \text { for } \mathfrak{a} \in X^{k} \Delta,
$$

where $f^{+}, f^{-}$are a pair of combinatorial functions satisfying

$$
f(a)=f^{+}(a)-f^{-}(a) \text { for } a \in X^{k} \omega .
$$

This definition is independent of the particular $f^{+}, f^{-}$used in (12), provided only that they satisfy (13). However in application we shall always use the decomposition (3) of [6]. In Definition 3 of [6] we extended every function $f: X^{k} \omega^{*} \rightarrow \omega^{*}$ to a function $f_{\Delta^{*}}: \times^{k} \Delta^{*} \rightarrow \Delta^{*}$ by

$$
f_{\Delta} \cdot\left(\mathfrak{a}-{ }^{*} \mathfrak{b}\right)=f_{\Delta}\left(\mathfrak{a}_{0}, \mathfrak{b}_{0}, \ldots, \mathfrak{a}_{k-1}, \mathfrak{b}_{k-1}\right) \text { for } \mathfrak{a}, \mathfrak{b} \in X^{k} \Delta
$$

where $f: X^{2 k} \omega \rightarrow \omega^{*}$ is extended as in (12), and is defined by

$$
f\left(a-{ }^{*} b\right)=f\left(a_{0}, b_{0}, \ldots, a_{k-1}, b_{k-1}\right) \text { for } a, b \in X^{k} \omega .
$$

It is natural to ask when (12) gives $f_{\Delta}: \times^{k} \Delta \rightarrow \Delta$. Note that $f: X^{k} \omega \rightarrow \omega$ is a necessary condition for this to happen, and that $f$ be combinatorial is a sufficient condition, since in the latter case $f=f^{+}$and the result follows by Theorem 5 of [5]. In this section we shall characterize the widest class of functions, each of which when extended by (12) always assumes values in $\Delta$. The extension (14) will be useful in this task.

If $f: X^{k} \omega \rightarrow \omega$ and $m \in X^{k} \omega$ define $f^{(m)}: X^{k} \omega \rightarrow \omega$ by

$$
f^{(m)}(a)=f\left(a+{ }^{*} m\right) \text { for } a \in \times^{k} \omega .
$$

We call $f: X^{k} \omega \rightarrow \omega$ eventually combinatorial (cf. [12]) if for some $m \in X^{k} \omega$ the function $f^{(m)}$ is combinatorial.

$$
\begin{aligned}
& \text { Let } h: I \rightarrow \omega \text { where } I \subseteq k \in \omega,|k-I|=t>0 \text { and } \\
& j: t \sim k-I \text { enumerates } k-I \text { in ascending order. }
\end{aligned}
$$

With $h$ and $k$ we associate a function $h_{k}^{*}: X^{t} \Gamma \rightarrow X^{k} \Gamma$ as follows. If $a \in X^{t} \Gamma$ let $h_{k}^{*}(\mathfrak{a})=\mathfrak{b} \in X^{k} \Gamma$ where $\mathfrak{b}_{i}=h(i)$ for $i \in I$ and $\mathfrak{b}_{j(i)}=\mathfrak{a}_{i}$ for $i<t$. A function $f: X^{k} \omega \rightarrow \omega$ is called almost combinatorial (cf. [12]) if for each $h$ satisfying (17) the composition $f \circ h_{k}^{*}$ is eventually combinatorial. Then we need the following

LEMMA 3. Let $f: X^{k} \omega \rightarrow \omega$ be combinatorial and let $h, t$ satisfy (17). If $m \in X^{k} \omega$, $\mathfrak{a} \in X^{t} \Delta^{\infty}$, and $\mathfrak{b}=h_{k}^{*}(\mathfrak{a})$ then $\left(f \circ h_{k}^{*}\right)^{(m)}$ is combinatorial and

$$
\left(f \circ h_{k}^{*}\right)_{\Delta}^{(m)}\left(\mathfrak{a}-{ }^{*} m\right)=f_{\Delta}(\mathfrak{b}) .
$$


Proof. Define combinatorial functions $g_{i}: \times^{t} \omega \rightarrow \omega$ for $i<k$ by $g_{i}(a)=h(i)$ for $i \in I$ and $g_{j(i)}(a)=a_{i}$ for $i<t$. This implies that

$$
\left(f \circ h_{k}^{*}\right)^{(m)}(a)=\left(f \circ\left(g_{0}, \ldots, g_{k-1}\right)\right)(a+* m)
$$

for $a \in X^{t} \omega$. The right side of (18) is combinatorial by Theorem 3 of [5], and hence by (8), formula (18) will identically hold when extended to $\Delta$. By Theorem 4 of [5] the extensions to $\Delta$ of constants, plus, and projections are the corresponding constants, plus, and projections. Thus $\left\langle g_{0, \Delta}(\mathfrak{a}), \ldots, g_{k-1, \Delta}(\mathfrak{a})\right\rangle=h_{k}^{*}(\mathfrak{a})=\mathfrak{b}$. Now evaluate (18) at $\mathfrak{a}-{ }^{*} m$ to obtain our result. Q.E.D.

THEOREM 3. Let $f: X^{k} \omega \rightarrow \omega$. (i) if $f$ is eventually combinatorial then

$$
f_{\Delta}: \times^{k} \Delta^{\infty} \rightarrow \Delta
$$

and (ii) if $f$ is almost combinatorial then $f_{\Delta}: X^{k} \Delta \rightarrow \Delta$.

Proof. (i) follows by specializing the proof of (ii). For (ii) let $\mathfrak{b} \in X^{k} \Delta-X^{k} \omega$. Take $I=\left\{i<k: \mathfrak{b}_{i} \in \omega\right\}$ and define $h: I \rightarrow \omega$ by $h(i)=\mathfrak{b}_{i}$ for $i \in I$. Let $t$ satisfy (17) and choose $\mathfrak{a} \in X^{t} \Delta^{\infty}$ such that $h_{k}^{*}(\mathfrak{a})=\mathfrak{b}$. Since $f$ is almost combinatorial there is an $m \in X^{t} \omega$ for which $\left(f \circ h_{k}^{*}\right)^{(m)}$ is combinatorial. Then by (13)

$$
\left(f \circ h_{k}^{*}\right)^{(m)}(a)+\left(f^{-} \circ h_{k}^{*}\right)^{(m)}(a)=\left(f^{+} \circ h_{k}^{*}\right)^{(m)}(a)
$$

for $a \in X^{t} \omega$, and hence by (8), formula (19) will identically hold when extended to $\Delta$. We evaluate (19) at $\mathfrak{a}-{ }^{*} m$ and use Lemma 3 to get $\left(f \circ h_{k}^{*}\right)_{\Delta}^{(m)}\left(\mathfrak{a}-{ }^{*} m\right)+f_{\Delta}^{-}(\mathfrak{b})$ $=f_{\Delta}^{+}(\mathfrak{b})$. But $\left(f \circ h_{k}^{*}\right)_{\Delta}^{(m)}\left(\mathfrak{a}-{ }^{*} m\right) \in \Delta$. Thus $f_{\Delta}^{-}(\mathfrak{b}) \leqq f_{\Delta}^{+}(\mathfrak{b})$ and hence by (12), $f_{\Delta}(\mathfrak{b}) \in \Delta$. Q.E.D.

Lemma 4. If $f: X^{k} \omega^{*} \rightarrow \omega^{*}$ then $f_{\Delta^{*}} \uparrow X^{k} \Delta=\left(f \uparrow \times^{k} \omega\right)_{\Delta}$.

Proof. Let $\mathfrak{a} \in X^{k} \Delta$ and observe that by (15)

$$
\left(f \uparrow \times^{k} \omega\right)(a)=\tilde{f}\left(a_{0}, 0, \ldots, a_{k-1}, 0\right) \text { for } a \in \times^{k} \omega .
$$

By (8), (13) and (12), formula (20) will identically hold when extended to $\Delta$. Now evaluate (20) at $\mathfrak{a}$ and use (14) to obtain our result. Q.E.D.

We call $f: X^{k} \omega \rightarrow \omega$ eventually constant if for some $m \in X^{k} \omega, f^{(m)}$ is a constant function. Then we have

THEOREM 4. Let $f: X^{n} \omega \rightarrow \omega$ and $f_{i}: X^{k} \omega \rightarrow \omega$ for $i<n$. Then (i) if $f$ is almost combinatorial and the $f_{i}$ are eventually combinatorial then the composition $g=f \circ\left(f_{0}, \ldots, f_{n-1}\right)$ is eventually combinatorial and $g_{\Delta^{\infty}}=f_{\Delta} \circ\left(f_{0, \Delta^{\infty}}, \ldots, f_{n-1, \Delta^{\infty}}\right)$ and (ii) if $f$ and the $f_{i}$ are almost combinatorial then the composition $g=$ $f \circ\left(f_{0}, \ldots, f_{n-1}\right)$ is almost combinatorial (cf. [12]), and $g_{\Delta}=f_{\Delta} \circ\left(f_{0, \Delta}, \ldots, f_{n-1, \Delta}\right)$.

Proof of (i). Let $I=\left\{i<n: f_{i}\right.$ is eventually constant $\}$ and let $h(i)=$ the constant value that $f_{i}$ eventually assumes for $i \in I$. If $I=n$ then $g$ is eventually constant, á fortiori eventually combinatorial. Thus suppose that $I \subseteq n,|n-I|=t>0$ and $j: t \sim n-I$ enumerates $n-I$ in ascending order. Now $f$ is almost combinatorial so 
that for some $m^{\prime} \in X^{t} \omega$ the function $f^{\prime}=\left(f \circ h_{n}^{*}\right)^{\left(m^{\prime}\right)}$ is combinatorial. Choose $m \in X^{k} \omega$ such that (a) each $f_{i}^{(m)}$ is combinatorial, (b) $f_{i}^{(m)}(a)=h(i)$ for $i \in I$, and (c) $f_{j(i)}^{(m)}(a) \geqq m_{i}^{\prime}$ for $i<t$. Note that in case (c) the functions $f_{i}^{\prime}(a)=f_{j(i)}^{(m)}(a)-m_{i}^{\prime}$ are combinatorial. Then

$$
\begin{aligned}
g^{(m)} & =\left(f \circ\left(f_{0}, \ldots, f_{n-1}\right)\right)^{(m)}=f \circ\left(f_{0}^{(m)}, \ldots, f_{n-1}^{(m)}\right) \\
& =\left(f \circ h_{n}^{*}\right) \circ\left(f_{j(0)}^{(m)}, \ldots, f_{j(t-1)}^{(m)}\right)=f^{\prime} \circ\left(f_{0}^{\prime}, \ldots, f_{t-1}^{\prime}\right) .
\end{aligned}
$$

It then follows by Theorem 3 of [5] that $g^{(m)}$ is combinatorial since the primed functions are. Thus $g$ is eventually combinatorial. For the second part of (i) let $f^{\prime}: \times^{n} \omega^{*} \rightarrow \omega^{*}$ be defined by $f^{\prime}(a)=f(a)$ for $a \in X^{n} \omega$ and $f^{\prime}(a)=0$ otherwise so that $g=f^{\prime} \circ\left(f_{0}, \ldots, f_{n-1}\right)$. Now by Lemma 4(i) of [6] $g_{\Delta}=f_{\Delta^{*}}^{\prime} \circ\left(f_{0, \Delta}, \ldots, f_{n-1, \Delta}\right)$. Our result follows from the first part of Theorem 3, and Lemma 4, by restricting arguments. Q.E.D.

Proof of (ii). Let $h, I, t$, and $j$ be any objects satisfying (17). Then $g \circ h_{k}^{*}=$ $f \circ\left(\left(f_{0} \circ h_{k}^{*}\right), \ldots,\left(f_{n-1} \circ h_{k}^{*}\right)\right)$. Since $f$ is almost combintorial and each $f_{i} \circ h_{k}^{*}$ is eventually combinatorial, $g \circ h_{k}^{*}$ is eventually combinatorial by the first part of Theorem 4(i). Hence $g$ is almost combinatorial. The second part of (ii) follows in exactly the same way as the second part of (i). Q.E.D.

If $\mathfrak{A}$ is a sentence of $L R$ and $\Sigma \subseteq \Delta$ let $\mathfrak{A}_{\Sigma}$ be the interpretation obtained by allowing variables to range of $\Sigma$, by letting functors denote extensions to $\Sigma$ of fixed almost combinatorial functions, and by letting predicates denote extensions to $\Sigma$ (by Definition 1) of fixed relations over $\omega$.

If $\mathfrak{A}$ is a universally quantified Horn sentence we have

THEOREM 5. If $\mathfrak{A}_{\omega}$ then $\mathfrak{A}_{\Delta}$.

Proof. Once we have taken care of atomic formulas the result will follow by Theorem 2. Use Theorem 4 to collapse compositions, and then Definition $1^{\prime}$ to bring atomic formula to the form $f_{0, \Sigma}(\mathfrak{a})=f_{1, \Sigma}(\mathfrak{a})$ for almost combinatorial $f_{0}, f_{1}$. By (12) and (13) such formula may be replaced by $f_{0, \Sigma}^{+}(\mathfrak{a})+f_{1, \Sigma}^{-}(\mathfrak{a})=f_{1, \Sigma}^{+}(\mathfrak{a})+f_{0, \Sigma}^{-}(\mathfrak{a})$. Since by our previous theorems these replacements are valid for $\Sigma=\omega$ and $\Sigma=\Delta$ our result follows. Q.E.D.

Again the remark following Theorem 2 is applicable in this case. It is also possible to formulate a version of Thesiem 5 where we let functors denote arbitrary functions $f: X^{k} \omega \rightarrow \omega$. Care must be taken however not to violate our closure conditions by entering $\Delta^{*}$. Whenever we use such a version, it will always be for a specific sentence where it is clear that no violation occurs.

If $R \subseteq X^{k+1} \Gamma, \mathfrak{a} \in X^{k} \Gamma$ and $\mathfrak{b} \in \Gamma$ write $R(\mathfrak{a}, \mathfrak{b})$ for $R\left(\mathfrak{a}^{\wedge}\langle\mathfrak{b}\rangle\right)$. We say $R$ is functional if for every $\mathfrak{a}$ there is at most one $\mathfrak{b}$ such that $R(\mathfrak{a}, \mathfrak{b})$. There is associated with every functional relation $R$ a function $r$ (and conversely) which is given by

$$
r(\mathfrak{a})=\mathfrak{b} \quad \text { iff } R(\mathfrak{a}, \mathfrak{b}) \quad \text { for } \mathfrak{a} \in X^{k} \Gamma, \mathfrak{b} \in \Gamma
$$


We use corresponding upper (lower) case letters to denote the associated relation (function) respectively, and call $R$ combinatorial etc. if its associated function is.

THEOREM 6. If $R \subseteq X^{k+1} \omega$ then (i) $R$ is functional if and only if $R_{\Delta}$ is functional, (ii) if $R$ is eventually combinatorial then $\left(\forall \mathfrak{a} \in X^{k} \Delta^{\infty}\right)(\exists \mathfrak{b} \in \Delta) R_{\Delta}(\mathfrak{a}, \mathfrak{b})$, in fact $\left(\forall \mathfrak{a} \in X^{k} \Delta^{\infty}\right) R_{\Delta}\left(\mathfrak{a}, r_{\Delta}(\mathfrak{a})\right)$, and (iii) if $R$ is almost combinatorial then $\left(\forall \mathfrak{a} \in X^{k} \Delta\right)$ $(\exists \mathfrak{b} \in \Delta) R_{\Delta}(\mathfrak{a}, \mathfrak{b})$, in fact $\left(\forall \mathfrak{a} \in X^{k} \Delta\right) R_{\Delta}\left(\mathfrak{a}, r_{\Delta}(\mathfrak{a})\right)$. ( $r$ associated with $R$ in (ii) and (iii)).

Proof. (i) follows from theorem 2 by a Horn sentence. From (21)

$$
\left(\forall \mathfrak{a} \in X^{k} \Sigma\right)(\forall \mathfrak{b} \in \Sigma)\left(r_{\Sigma}(\mathfrak{a})=\mathfrak{b} \text { iff } R_{\Sigma}(\mathfrak{a}, \mathfrak{b})\right)
$$

holds for $\Sigma=\omega$ and hence by the remark following Theorem 5 and the totality of $r$, for $\Sigma=\Delta$. Then (ii) and (iii) follow by Theorem 3. Q.E.D.

4. Functions as relations. In this section we shall try to find a converse to Theorems 3 and 6. Obviously we cannot prove that $f_{\Delta}: X^{k} \Delta \rightarrow \Delta$ implies that $f$ is almost combinatorial; for if so we could add AC to our theory, forcing $\Delta=\omega$, and thus prove that every function $f$ is almost combinatorial. Rather we shall find an extension of $\mathbb{S}^{\circ}$, relatively consistent with $\mathbb{S}^{\circ}$, though not with $\mathbb{S}^{\circ}+\mathrm{AC}$, in which the desired converse holds. Before doing so we need the following

Lemma 5. Let $M \in X^{k} V$ each component of which is infinite and let

$$
F=\left\{A \in \times^{k} Q: A \subseteq{ }^{*} M\right\} .
$$

If $\Phi: F \rightarrow Q$ satisfies for every $A, A^{\prime} \in F$, (i) $A \sim^{*} A^{\prime}$ implies $\Phi(A) \sim \Phi\left(A^{\prime}\right)$, and (ii) $\Phi\left(A \cap \cap^{*} A^{\prime}\right)=\Phi(A) \cap \Phi\left(A^{\prime}\right)$, then $f: \times^{k} \omega \rightarrow \omega$ defined by $f\left(|A|^{*}\right)=|\Phi(A)|$ for $A \in F$, is a combinatorial function.

Proof. (ii) implies that we can define a quasi-inverse function $\Phi^{-1}: \cup \rho \Phi \rightarrow F$ such that $x \in \Phi(A)$ iff $\Phi^{-1}(x) \subseteq^{*} A$, for $A \in F$. Then by (i) $\Phi$ is the restriction to $F$ of some combinatorial operator. Since $F$ is a frame we can define $\Phi^{F}: F \rightarrow Q$ by (10) and prove an exact analogue of Lemma 2, i.e., for every $A, B \in F$, (a) $A \neq B$ implies $\Phi^{F}(A) \cap \Phi^{F}(B)=\varnothing$, and (b) $\Phi(B)=\bigcup\left\{\Phi^{F}(A) ; A \in F \wedge A \subseteq * B\right\}$. Also, as in the proof of Theorem 1 we can use (i) and prove by induction that (c) $A \sim^{*} B$ implies $\Phi^{F}(A) \sim \Phi^{F}(B)$. Define $c\left(|A|^{*}\right)=\left|\Phi^{F}(A)\right|$ for $A \in F$. Since $M$ is infinite in each component, for every $b \in \times^{k} \omega$ we can find a $B \in F$ with $b=|B|^{*}$. Then

$$
f(b)=|\Phi(B)|=\Sigma c(i)\left(\begin{array}{l}
b \\
i
\end{array}\right)
$$

by (a), (b), and (c) which proves that $f$ is combinatorial. Q.E.D.

To our language of set theory add an individual constant $\boldsymbol{K}$ and a functor $\boldsymbol{\sigma}$ and let $\mathfrak{S}$ be the theory obtained from $\mathfrak{F S}^{\circ}$ by taking as additional axioms

(23) $K \subseteq \mathfrak{B}(\omega)$ is dense in the canonical ordering $\prec$ of $\mathfrak{B}(\omega)$.

(24) $\boldsymbol{\sigma}: \boldsymbol{V} \rightarrow \mathfrak{P}_{\omega}(\boldsymbol{K})$ and reduces to the identity on $\mathfrak{P}_{\omega}(\boldsymbol{K})$.

(25) If $x \in y$ and $y$ is well orderable then $\sigma(x) \subseteq \sigma(y)$.

(26) If $\left(\forall x_{0}, \ldots, x_{k-1}\right)\left(\exists ! x_{k}\right) \varphi$ then $\sigma\left(x_{k}\right) \subseteq \bigcup\left\{\sigma\left(x_{j}\right): j<k\right\}$, 
where $\varphi$ is any formula of set theory possibly involving $\boldsymbol{K}$ and $\boldsymbol{\sigma}$ as parameters. From [10] as reported in [2], and from [7], if $\mathbb{S}^{0}$ is consistent then so is $\mathfrak{F}$. Let us first observe that $K$ is an infinite Dedekind set. For $K$ is infinite by (23), and if $A \subseteq K$ is well orderable then $x \in A$ implies $\{x\}=\sigma(x) \subseteq \sigma(A)$ by (24)-(26) and hence that $A \subseteq \sigma(A) \in Q$, i.e., $A$ is finite. For $a, b \in K$ with $a \prec b$ let

$$
(-\infty, b)=\{x \in \boldsymbol{K}: x \prec b\}, \quad(a, b)=\{x \in \boldsymbol{K}: a \prec x \prec b\},
$$

and $(a, \infty)=\{x \in K: a \prec x\}$. If $A \in \mathfrak{P}_{\omega}(\boldsymbol{K})$ and $A=\left\{a_{0}, \ldots, a_{k-2}\right\}$ with $a_{0} \prec \cdots$ $\prec a_{k-2}$ let $\boldsymbol{K}^{A}=\left\langle\boldsymbol{K}_{0}^{A}, \ldots, \boldsymbol{K}_{k-1}^{A}\right\rangle$ where $\boldsymbol{K}_{0}^{A}=\left(-\infty, a_{0}\right), \boldsymbol{K}_{j+1}^{A}=\left(a_{j}, a_{j+1}\right)$ for $j<k-2$, and $\boldsymbol{K}_{k-1}^{A}=\left(a_{k-2}, \infty\right)$. Finally let $\mathfrak{f}^{A}=\left|\boldsymbol{K}^{A}\right|^{*}$ and observe that since each $\boldsymbol{K}_{j}^{A}$ is infinite by (23), and since $K$ is Dedekind, we have $\mathfrak{f}^{A} \in X^{k} \Delta^{\infty}$. Then the main result of this section is

$\mathfrak{S}$-THEOREM 7. Let $R \subseteq X^{k+1} \omega$ be functional and let $r$ be its associated function. If $(\exists \mathfrak{m} \in \Delta) R_{\Delta}\left(\mathfrak{f}^{A}, \mathfrak{m}\right)$ then every extension of $r$ to a function $r^{\prime}: X^{k} \omega \rightarrow \omega$ is eventually combinatorial (note that if $\delta r=X^{k} \omega$ then $r$ is eventually combinatorial).

Proof. By hypothesis there is an $\mathfrak{m} \in \Delta$ such that $R_{\Delta}\left(\mathfrak{f}^{A}, \mathfrak{m}\right)$ and hence a set $M$ with $\mathfrak{m}=|M|$ and an $R$-frame $F$ such that $\left\langle K^{A}, M\right\rangle \in \mathscr{A}(F)$. For convenience we write $\langle a, b\rangle$ instead of $a^{\wedge}\langle b\rangle$. Choose $S \in X^{k} \boldsymbol{Q}$ such that $\sigma(F) \cap K_{j}^{A} \subseteq S_{j} \subseteq K_{j}^{A}$ for $j<k$ and let $G=\left\{B \in \times^{k} Q: S \subseteq^{*} B \subseteq \subseteq^{*} K^{A}\right\}$. Now for each $B \in G,\langle B, \varnothing\rangle$ $\subseteq^{*}\left\langle K^{A}, M\right\rangle \in \mathscr{A}(F)$ so that by the remark following (4) $\gamma_{F}(\langle B, \varnothing\rangle)=\left\langle B^{\prime}, C\right\rangle \in F$ where $B \subseteq \subseteq^{*} B^{\prime} \in G$ and $C \in \mathfrak{P}_{\omega}(M)$. I claim that $B=B^{\prime}$; for otherwise there is a $j<k$ and an $x \in B_{j}^{\prime}-B_{j} \subseteq K_{j}^{A}$. Then $\{x\}=\sigma(x) \subseteq \sigma\left(B_{j}^{\prime}\right) \subseteq \sigma\left(B^{\prime}\right) \subseteq \sigma(F) \cup \sigma(B)=$ $A \cup \cup\left\{B_{i}: i<k\right\}$ by (23)-(26). But $x \in K_{j}^{A}$ and so $x \in B_{j}$ which is a contradiction. Thus $\langle B, C\rangle \in F$. Further I claim that $C$ is uniquely determined by $B$; for otherwise there is a $C^{\prime}$ such that $\left\langle B, C^{\prime}\right\rangle \in F$ and hence by (1) $\left\langle B, C \cap C^{\prime}\right\rangle \in F$. But $R$ is functional so that $C=C \cap C^{\prime}=C^{\prime}$. For $B \in G$ define $\Psi(B)=C$ where $C$ is the unique element satisfying $\langle B, C\rangle \in F$. Then $\Psi$ has the following properties. (i) If $B, B^{\prime} \in G$ and $B \sim \sim^{*} B^{\prime}$ then $\Psi(B) \sim \Psi^{\prime}\left(B^{\prime}\right)$, follows from the fact that $R$ is functional. Also, if $B, B^{\prime} \in G$ then $\langle B, \Psi(B)\rangle \in F$ and $\left\langle B^{\prime}, \Psi\left(B^{\prime}\right)\right\rangle \in F$ so that by (1), $\left\langle B \cap^{*} B^{\prime}\right.$, $\left.\Psi(B) \cap \Psi\left(B^{\prime}\right)\right\rangle \in F$ as well. But $B \cap^{*} B^{\prime} \in G$ and hence $\left\langle B \cap^{*} B^{\prime}, \Psi\left(B \cap * B^{\prime}\right)\right\rangle \in F$. This implies that (ii) if $B, B^{\prime} \in G$ then $\Psi\left(B \cap^{*} B^{\prime}\right)=\Psi(B) \cap \Psi\left(B^{\prime}\right)$. Let

$$
H=\left\{B \in \times^{*} Q: B \subseteq K^{A} \wedge B \cap * S=\varnothing\right\}
$$

and define $\Phi(B)=\Psi\left(B \cup^{*} S\right)$ for $B \in H$. From the corresponding properties of $\Psi$ we have for $B, B^{\prime} \in H$, (i) if $B \sim^{*} B^{\prime}$ then $\Phi(B) \sim \Phi\left(B^{\prime}\right)$, and (ii) $\Phi\left(B \cap^{*} B^{\prime}\right)$ $=\Phi(B) \cap \Phi\left(B^{\prime}\right)$. Define $f: \times^{k} \omega \rightarrow \omega$ by $f(|B|)=|\Phi(B)|$ for $B \in H$ and note that by Lemma $5 f$ is combinatorial. Thus if $B \in H$ then $\langle B \cup * S, \Phi(B)\rangle \in F$. If we take $s=|S|$ this implies that $R\left(a+{ }^{*} s, f(a)\right)$ for all $a \in X^{k} \omega$. This gives $r^{(s)}=f$ for the associated function $r$, making any extension $r^{\prime}: X^{k} \omega \rightarrow \omega$ eventually combinatorial. Q.E.D.

Let $h, I, k, t$, and $j$ satisfy (17). If $R \subseteq X^{k} \Gamma$ define the $h$-specification $\mathscr{S}_{h} R$ 
$=\left\{\mathfrak{a} \in X^{t} \Gamma: h_{k}^{*}(\mathfrak{a}) \in R\right\}$. Then by using the same method as in the proof of Lemma 3 it can easily be shown that

LEMma 6. If $R \subseteq X^{k} \omega$ and $h$ satisfies (17) then $\left(\mathscr{S}_{h} R\right)_{\Delta}=\mathscr{S}_{h}\left(R_{\Delta}\right)$.

$\mathfrak{S}$-THEOREM 8. If $R \subseteq X^{k+1} \omega$ and $\left(\forall \mathfrak{a} \in X^{k} \Delta\right)(\exists ! \mathfrak{b} \in \Delta) R_{\Delta}(\mathfrak{a}, \mathfrak{b})$ then $R$ is almost combinatorial.

Proof. $R_{\Delta}$ is functional and hence by Theorem 6 so is $R$. The domain of the function $r$ associated with $R$ is $X^{k} \omega$. For otherwise there is an $m \in X^{k} \omega$ such that $(\forall b \in \omega) \sim R(m, b)$ and hence by Theorem 2 a corresponding result (with the same $m$ as parameter) would hold in $\Delta$. Now consider any $h$ and $t$ which satisfy (17). By specialization of arguments $\left(\forall \mathfrak{a} \in X^{t} \Delta^{\infty}\right)(\exists \mathfrak{b} \in \Delta) \mathscr{S}_{h}\left(R_{\Delta}\right)(\mathfrak{a}, \mathfrak{b})$, and hence by Lemma 6 the same result would hold with $\mathscr{S}_{h}\left(R_{\Delta}\right)$ replaced by $\left(\mathscr{S}_{h} R\right)_{\Delta}$. By Theorem 7 this implies that $\mathscr{S}_{h} R$ is eventually combinatorial. But $\mathscr{S}_{h} R$ is the relation associated with $r \circ h_{k}^{*}$ which is also eventually combinatorial. Thus $r$ is almost combinatorial. Q.E.D.

An immediate consequence of (22) and Theorem 7 and 8 is

Se-TheOREM 9. Let $f: \times^{k} \omega \rightarrow \omega$. (i) if $f_{\Delta}\left(\mathfrak{t}^{A}\right) \in \Delta$ then $f$ is eventually combinatorial, á fortiori if $f_{\Delta}: \times^{k} \Delta^{\infty} \rightarrow \Delta$ then $f$ is eventually combinatorial, and (ii) is $f_{\Delta}: X^{k} \Delta \rightarrow \Delta$ then $f$ is almost combinatorial.

By using the relativization technique of [7] we can obtain

MetaCorollary 1. Let $f: X^{k} \omega \rightarrow \omega$ be definable by a two function quantifier form in the analytic hierarchy. If we can prove in $\mathrm{ss}^{0}$ that $f_{\Delta}: X^{k} \Delta^{\infty} \rightarrow \Delta$ $\left(f: X^{k} \Delta \rightarrow \Delta\right)$ then we can prove in $\mathbb{S}$ that $f$ is an eventually (almost) combinatorial function.

We call $\mathfrak{t} \in X^{k} \Delta$ strongly universal if for every $f: X^{k} \omega \rightarrow \omega, f_{\Delta}(\mathfrak{t}) \in \Delta$ implies that $f$ is eventually combinatorial, and (weakly) universal if $f_{\Delta}(\mathfrak{t})=0$ implies that $f$ is eventually equal to 0 .

THEOREM 10. (i) If $\mathfrak{t} \in X^{k} \Delta$ is universal, strong or weak, then $\mathfrak{t} \in X^{k} \Delta^{\infty}$, (ii) if $\mathfrak{t}$ is strongly universal then is weakly universal, and (iii) if $\mathfrak{t} \in X^{k} \Delta$ is universal, $R \subseteq X^{k} \omega$, and $R_{\Delta}(\mathfrak{f})$ then $\times^{k}(\omega-m) \subseteq R$ for some $m \in \omega$ (in this case we say $R$ eventually holds).

Proof. We only prove the more difficult case of (i). Suppose that is strongly universal but $\mathfrak{t} \notin X^{k} \Delta^{\infty}$. Let $I=\left\{i<k: \mathfrak{f}_{i} \in \omega\right\}$ and define $h: I \rightarrow \omega$ by $h(i)=\mathfrak{f}_{i}$ for $i \in I$. If $|k-I|=t$ we may choose some $\mathfrak{t}^{\prime} \in X^{t} \Delta^{\infty}$ such that $h_{k}^{*}\left(\mathfrak{t}^{\prime}\right)=\mathfrak{t}$. Now by hypothesis $t<k$ so we can find a function $f: X^{k} \omega \rightarrow \omega$ not eventually combinatorial for which $f \circ h_{k}^{*}$ is combinatorial. Since we can easily remove the hypothesis that $f$ be combinatorial in Lemma 3 (by expressing $f=f^{+}-f^{-}$) it follows that $f_{\Delta}(\mathfrak{t})=\left(f \circ h_{k}^{*}\right)_{\Delta}\left(\mathfrak{t}^{\prime}\right) \in \Delta$ which contradicts the fact that $\mathfrak{t}$ is strongly universal. To prove (ii) suppose that $\mathrm{t} \in X^{k} \Delta$ is strongly universal, that $f: X^{k} \omega \rightarrow \omega$, and that 
$f_{\Delta}(\mathfrak{t})=0 \in \Delta$. Thus $f$ is eventually combinatorial. Since eventually combinatorial functions are eventually monotone, there is an $m \in X^{k} \omega$ such that $f^{(m)}$ is combinatorial and either $f^{(m)}$ is identically equal to 0 or $\left(\forall a \in X^{k} \Sigma\right) f_{\Sigma}^{(m)}(\mathfrak{a}) \neq 0$ holds for $\Sigma=\omega$ and hence by Theorem 2 for $\Sigma=\Delta$. Then in the latter case we could use Lemma 3 and the fact that $\mathfrak{t} \in X^{k} \Delta^{\infty}$ to obtain $f_{\Delta}(\mathfrak{t})=f_{\Delta}^{(m)}\left(\mathfrak{t}-{ }^{*} m\right) \neq 0$ which contradicts our hypothesis. Hence $f$ is eventually equal to 0 which implies that $\mathfrak{t}$ is weakly universal. To prove (iii) suppose that $\mathfrak{f} \in X^{k} \Delta$ is universal, that $R \subseteq X^{k} \omega$, and that $R_{\Delta}(\mathfrak{t})$. Let $f: X^{k} \omega \rightarrow\{0,1\}$ be the characteristic of $R$, i.e., $f(a)=0$ for $a \in R$ and $f(a)=1$ for $a \in X^{k} \omega-R$. Thus $\left(\forall \mathfrak{a} \in X^{k} \Sigma\right)\left(R_{\Sigma}(\mathfrak{a}) \rightarrow f_{\Sigma}(\mathfrak{a})=0\right)$ holds for $\Sigma=\omega$ and hence by the remark following Theorem 5 for $\Sigma=\Delta$. But then $f_{\Delta}(\mathfrak{f})=0$, so $f$ is eventually 0 which implies $X^{k}(\omega-m) \subseteq R$ for some $m \in \omega$. Q.E.D.

Consider the following extensions of our basic set theory (5) ${ }^{0}$.

$\mathfrak{B S}^{\circ}(\Delta)=\mathfrak{S}^{0}+$ there exists an infinite $\mathfrak{a} \in \Delta$

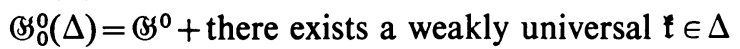

SSI $_{1}^{0}(\Delta)=\mathbb{S S}^{0}+$ there exists a strongly universal $\mathfrak{t} \in \Delta$.

Since $\mathfrak{H}$ is consistent relative to $\mathscr{S S}$, Theorems 9 and 10 imply that each of the above theories is consistent relative to $\$$ S. At the present time we have no knowledge as to their interrelationships, except as given by Theorem 10. Consequences of these axioms will be investigated in the next section. Before doing so let us make the following observation. Let $\mathfrak{A}$ be a single equation between terms built up from functors for + and $\cdot$, and the variables $\mathfrak{a}_{0}, \ldots, \mathfrak{a}_{k-1}, \mathfrak{b}$. Then by Metacorollary 1 we can prove $\left(\forall \mathfrak{a} \in X^{k} \Delta\right)(\exists ! \mathfrak{b} \in \Delta) \mathfrak{A}_{\Delta}$ in $\mathscr{B S}^{0}$ iff we can prove in $\mathbb{S}$ that a corresponding sentence holds in $\omega$, and moreover that its uniquely defined Skolem function is almost combinatorial. Thus we see that the answer to a classical question about the + , t theory of cardinal numbers involves the notion of almost combinatorial function. We hope that this point will convince the reader of the naturalness of combinatorial functions in axiomatic set theory.

5. Cardinality of extensions. Using the fact that combinatorial functions are eventually monotone as in the proof of Theorem 10(ii) we easily obtain

LEMMA 7. If $f: \omega \rightarrow \omega$ is a nonconstant combinatorial function then (i) $f_{\Delta}: \Delta^{\infty} \rightarrow \Delta^{\infty}$, and (ii) $f_{\Delta}\left\lceil\Delta^{\infty}\right.$ is one one on its domain.

Let $b=|\Delta|$ and $c=2^{x_{0}}$. These cardinalities are related by

THEOREM 11. (i) If $0<k<\omega$ then $\delta^{k}=\delta$ (here $k$ is used as an exponent), and (ii) if $\Delta^{\infty} \neq \varnothing$ then $\mathfrak{c} \leqq \delta\left(\right.$ so $\mathfrak{d}=\left|\Delta^{\infty}\right|, \delta=\mathfrak{d}$, etc. $)$.

Proof. From [5] we know of the existence of a one one combinatorial function $j: X^{k} \omega \rightarrow \omega$. Since one oneness can be expressed by a Horn sentence $j_{\Delta}: X^{k} \Delta \rightarrow \Delta$ is also one one. Thus $\mathfrak{d}^{k} \leqq \mathfrak{d}$ and (i) follows by an application of the CantorBernstein theorem. We prove (ii) in the following way. Let $A$ be the rational numbers and $A^{\#}$ the real numbers conceived as lower halves of Dedekind cuts. 
Let $h: \omega \sim A$ and for $\alpha \in A^{\#}$ define functions $c^{\alpha}: \omega \rightarrow\{0,1\}$ by $c^{\alpha}(i)=1$ if $h(i) \in \alpha$ and $c^{\alpha}(i)=0$ if $h(i) \notin \alpha$. With each such $\alpha$ we associate a combinatorial function $f^{\alpha}: \omega \rightarrow \omega$ by taking $c^{\alpha}$ as its combinatorial coefficients. If $\alpha, \beta \in A^{\#}$ with $\alpha<\beta$ it is easy to see that for some nonconstant combinatorial $g: \omega \rightarrow \omega$ we have $f^{\alpha}(a)+g(a)$ $=f^{\beta}(a)$ for $a \in \omega$. By Theorem 2 this identity extends to $\Delta$ and by Lemma 7 if $\mathfrak{a} \in \Delta^{\infty}$ then $g_{\Delta}(\mathfrak{a}) \neq 0$ so that $f_{\Delta}^{\alpha}(\mathfrak{a})<f_{\Delta}^{\beta}(\mathfrak{a})$. Thus $\left\{f_{\Delta}^{\alpha}(\mathfrak{a}): \alpha \in A^{\#}\right\}$ is a subset of $\Delta$, in fact of $\Delta^{\infty}$, which has cardinality c. Q.E.D.

If we use the methods of [7], and replace combinatorial by permutational throughout the preceding proof, we can actually obtain Theorem 11 without using $\mathrm{AC}^{0}$. In this form Theorem 11(ii) is an unpublished result of Tarski. If $R, S \subseteq X^{k} \omega$ then either by using Theorem 2 or the basic frame concept it is easy to establish that

(27) if $R \subseteq S$ then $R_{\Delta} \subseteq S_{\Delta}$, and $(R \cap S)_{\Delta}=R_{\Delta} \cap S_{\Delta}$.

We call a relation $R \subseteq X^{k} \omega$ limited if there is an $m \in \omega$ such that $R \cap \times^{k}(\omega-m)$ $=\varnothing$, and otherwise unlimited. Thus $R$ is limited if and only if $X^{k} \omega-R$ eventually holds (cf. Theorem 10(iii)). A fundamental result concerning this notion, which was proved in [12], is that

if $R \subseteq X^{k} \omega$ is infinite then for some $I \subseteq k$ and

$h: I \rightarrow \omega$ the relation $\mathscr{S}_{h} R$ is unlimited.

THEOREM 12. Let $R, S \subseteq \times^{k} \omega$. (i) if $R-S$ is limited then $R_{\Delta^{\infty}}=(R \cap S)_{\Delta^{\infty}}$ and $S_{\Delta^{\infty}}=(R \cup S)_{\Delta^{\infty}}$, and (ii) if $R-S$ is finite then $R_{\Delta}-\times^{k} \omega=(R \cap S)_{\Delta}-\times^{k} \omega$ and $S_{\Delta}-\times^{k} \omega=(R \cup S)_{\Delta}-\times^{k} \omega$.

Proof. Suppose $R-S$ is limited. Then there is an $m \in \times{ }^{k} \omega$ such that

$$
\left(\forall \mathfrak{a} \in X^{k} \Sigma\right)\left((R \cup S)_{\Sigma}\left(\mathfrak{a}+{ }^{*} m\right) \rightarrow S_{\Sigma}\left(\mathfrak{a}+{ }^{*} m\right)\right)
$$

holds for $\Sigma=\omega$ and hence by Theorem 2 for $\Sigma=\Delta$. Thus $(R \cup S)_{\Delta^{\infty}} \subseteq S_{\Delta^{\infty}}$ with the converse inclusion following by (27). This gives $(R \cup S)_{\Delta^{\infty}}=S_{\Delta^{\infty}}$. Also $R_{\Delta^{\infty}} \subseteq R_{\Delta^{\infty}}$ $\cap(R \cup S)_{\Delta^{\infty}}=R_{\Delta^{\infty}} \cap S_{\Delta^{\infty}}=(R \cap S)_{\Delta^{\infty}}$ with the converse inclusion following by (27). This gives $(R \cap S)_{\Delta^{\infty}}=R_{\Delta^{\infty}}$. Suppose $R-S$ is finite. For any $\mathfrak{b} \in(R \cup S)_{\Delta}$ $-X^{k} \omega$ let $I=\left\{i<k: \mathfrak{b}_{i} \in \omega\right\}$ and define $h: I \rightarrow \omega$ by $h(i)=\mathfrak{b}_{i}$ for $i \in I$. If $|k-I|$ $=t>0$ pick $\mathfrak{a} \in X^{t} \Delta^{\infty}$ such that $h_{k}^{*}(\mathfrak{a})=\mathfrak{b}$. Then $\mathfrak{a} \in \mathscr{S}_{h}\left((R \cup S)_{\Delta}\right) \cap \times^{t} \Delta^{\infty}$ $=\left(\mathscr{S}_{h}(R \cup S)\right)_{\Delta} \cap \times^{t} \Delta^{\infty}=\left(\mathscr{S}_{h}(R \cup S)\right)_{\Delta^{\infty}}=\left(\mathscr{S}_{h} R \cup \mathscr{S}_{h} S\right)_{\Delta^{\infty}}=\left(\mathscr{S}_{h} S\right)_{\Delta^{\infty}} \subseteq\left(\mathscr{S}_{h} S\right)_{\Delta}$ $=\mathscr{S}_{h}\left(S_{\Delta}\right)$ by Lemma 6 , part (i) of this theorem, and the fact that $\mathscr{S}_{h} R-\mathscr{S}_{h} S$ is limited. Hence $\mathfrak{b}=h_{k}^{*}(\mathfrak{a}) \in S_{\Delta}-\times^{k} \omega$. Thus $(R \cup S)_{\Delta}-X^{k} \omega \subseteq S_{\Delta}-X^{k} \omega$ with the converse inclusion following by (27). This gives $(R \cup S)_{\Delta}-\times^{k} \omega=S_{\Delta}-\times^{k} \dot{\omega}$. We also have $R_{\Delta}-\times^{k} \omega \subseteq R_{\Delta} \cap\left((R \cup S)_{\Delta}-\times^{k} \omega\right)=R_{\Delta} \cap\left(S_{\Delta}-X^{k} \omega\right)=$ $\left(R_{\Delta} \cap S_{\Delta}\right)-X^{k} \omega=(R \cap S)_{\Delta}-X^{k} \omega$. Thus $R_{\Delta}-X^{k} \omega \subseteq(R \cap S)_{\Delta}-X^{k} \omega$ with the converse inclusion following by (27). This gives $(R \cap S)_{\Delta}-\times^{k} \omega=R_{\Delta}-\times^{k} \omega$. Q.E.D.

Let $\oplus$ denote symmetric difference. Then we have the following 
Corollary 2. Let $R, S \subseteq \times^{k} \omega$. (i) If $R$ is limited then $R_{\Delta^{\infty}}=\varnothing$, (ii) if $\times^{k} \omega-R$ is limited then $R_{\Delta^{\infty}}=\times^{k} \Delta^{\infty}$, (iii) if $R \oplus S$ is limited then $(R \cap S)_{\Delta^{\infty}}=(R \cup S)_{\Delta^{\infty}}$, (iv) if $R$ is finite then $R_{\Delta}=R$, (v) if $\times^{k} \omega-R$ is finite then $\times^{k} \Delta-R_{\Delta}=\times^{k} \omega-R$, then (vi) if $R \oplus S$ is finite then $(R \cap S)_{\Delta}-\times^{k} \omega=(R \cup S)_{\Delta}-\times^{k} \omega$.

(S) $^{\circ}(\Delta)$-THEOREM 13. Let $R, S \subseteq \times^{k} \omega$. (i) if $R-S$ is unlimited then

$$
\left|R_{\Delta^{\infty}}-(R \cap S)_{\Delta^{\infty}}\right|=\mathfrak{d} \text { and }\left|(R \cup S)_{\Delta^{\infty}}-S_{\Delta^{\infty}}\right|=\mathfrak{d}
$$

and

(ii) if $R-S$ is infinite then $\left|R_{\Delta}-(R \cap S)_{\Delta}\right|=\mathfrak{d}$ and $\left|(R \cup S)_{\Delta}-S_{\Delta}\right|=\mathfrak{\delta}$.

Proof. Suppose $R-S$ is unlimited. We can construct $k$ unary nonconstant combinatorial functions $f_{j}$, such that $f(a)=\left\langle f_{0}(a), \ldots, f_{k-1}(a)\right\rangle \in R-S$ for $a \in \omega$. This follows because we may choose values of $f_{j}(a+1)$ sufficiently larger than $f_{j}(a)$ so as to make the $f_{j}$ nonconstant combinatorial functions, and yet keep $f(a+1)$ $\in R-S$. Now $f(a) \in R-S$ can be expressed by a Horn sentence, and hence by Theorem $2 f_{\Delta}(\mathfrak{a})=\left\langle f_{0, \Delta}(\mathfrak{a}), \ldots, f_{k-1, \Delta}(\mathfrak{a})\right\rangle \in R_{\Delta}-S_{\Delta}$ for $\mathfrak{a} \in \Delta$. By Theorem 11, $\left|\Delta^{\infty}\right|=\mathfrak{b}$, and by Lemma 7 , if $\mathfrak{a}, \mathfrak{a}^{\prime} \in \Delta^{\infty}$ then $f_{j, \Delta}(\mathfrak{a}) \in \Delta^{\infty}$, and $f_{j, \Delta}(\mathfrak{a})=f_{j, \Delta}\left(\mathfrak{a}^{\prime}\right)$ implies $\mathfrak{a}=\mathfrak{a}^{\prime}$, so that $\left\{f_{\Delta}(\mathfrak{a}): \mathfrak{a} \in \Delta^{\infty}\right\} \subseteq R_{\Delta^{\infty}}-S_{\Delta^{\infty}}$ has cardinality $\mathfrak{b}$. However by (27) it is clear that $R_{\Delta^{\infty}}-S_{\Delta^{\infty}} \subseteq R_{\Delta^{\infty}}-(R \cap S)_{\Delta^{\infty}}$ and $R_{\Delta^{\infty}}-S_{\Delta^{\infty}} \subseteq(R \cup S)_{\Delta^{\infty}}-S_{\Delta^{\infty}}$. Since by Theorem 11 both sets to the right of the inclusion have cardinality at most $\delta$, our result (i) follows from the Cantor-Bernstein theorem. Now suppose that $R-S$ is infinite. Then by (28) there is an $I \subseteq k$ and $h: I \rightarrow \omega$ such that $\mathscr{S}_{h}(R-S)=\mathscr{S}_{h} R-\mathscr{S}_{h} S$ is unlimited. If $|k-I|=t>0$ then $\left(\mathscr{S}_{h} R \cup \mathscr{S}_{h} S\right)_{\Delta^{\infty}}$ $-\left(\mathscr{S}_{h} S\right)_{\Delta^{\infty}}=\mathscr{S}_{h}\left((R \cup S)_{\Delta}-S_{\Delta}\right) \cap \times^{t} \Delta^{\infty} \quad$ and $\quad\left(\mathscr{S}_{h} R\right)_{\Delta^{\infty}}-\left(\mathscr{S}_{h} R \cap \mathscr{S}_{h} S\right)_{\Delta^{\infty}}=$ $\mathscr{S}_{h}\left(R_{\Delta}-(R \cap S)_{\Delta}\right) \cap \times^{t} \Delta^{\infty}$ both have cardinality $\delta$ by part (i) of this theorem. Hence $(R \cup S)_{\Delta}-S_{\Delta}$ and $R_{\Delta}-(R \cap S)_{\Delta}$ have cardinality at least $\mathrm{D}$ since $h_{k}^{*}$ is a one one function. Our result (ii) then follows as above from the Cantor-Bernstein theorem. Note that (ii) is trivial if $\Delta^{\infty}=\varnothing$ (and $\delta=\aleph_{0}$ ). Q.E.D.

BS $^{\circ}(\Delta)$-Corollary 3. Let $R, S \subseteq X^{k} \omega$. (i) If $R$ is unlimited then $\left|R_{\Delta^{\infty}}\right|=\mathfrak{D}$, (ii) if $\times^{k} \omega-R$ is unlimited then $\left|\times^{k} \Delta^{\infty}-R_{\Delta^{\infty}}\right|=\delta$, (iii) if $R \oplus S$ is unlimited then $\left|(R \cup S)_{\Delta^{\infty}}-(R \cap S)_{\Delta^{\infty}}\right|=\mathfrak{D}$, (iv) if $R$ is infinite then $\left|R_{\Delta}\right|=\mathfrak{D}$, (v) if $\times^{k} \omega-R$ is infinite then $\left|\times^{k} \Delta-R_{\Delta}\right|=\mathfrak{D}$, and (vi) if $R \oplus S$ is infinite then

$$
\left|(R \cup S)_{\Delta}-(R \cap S)_{\Delta}\right|=\mathfrak{\delta} .
$$

THEOREM 14. Let $R, R_{i} \subseteq \times^{k} \omega$ for $i<\omega$ such that $R \subseteq \bigcup\left\{R_{i}: i<\omega\right\}$. (i) If some $R-R_{j}$ is limited then $R_{\Delta^{\infty}} \subseteq \bigcup\left\{R_{i, \Delta^{\infty}}: i<\omega\right\}$, and (ii) if for every $I \subseteq k$ and $h: I \rightarrow \omega$ some $\mathscr{S}_{h}\left(R-R_{j}\right)$ is limited then $R_{\Delta} \subseteq \bigcup\left\{R_{i, \Delta}: i<\omega\right\}$.

Proof. If for some $j<\omega, R-R_{j}$ is limited then by Theorem 12 (i) $R_{\Delta^{\infty}}=$ $\left(R \cap R_{j}\right)_{\Delta^{\infty}} \subseteq R_{j, \Delta^{\infty}} \subseteq \bigcup\left\{R_{i, \Delta^{\infty}}: i<\omega\right\}$. This proves (i). For (ii) take $\mathfrak{b} \in R_{\Delta}$. If $\mathfrak{b} \in X^{k} \omega$ there is nothing to prove, so take $\mathfrak{b} \in R_{\Delta}-X^{k} \omega$. Let $I=\left\{i<k: \mathfrak{b}_{i} \in \omega\right\}$ and define $h: I \rightarrow \omega$ by $h(i)=\mathfrak{b}_{\mathfrak{i}}$ for $i \in I$. If $|k-I|=t>0$ pick $\mathfrak{a} \in X^{t} \Delta^{\infty}$ such that 
$h_{k}^{*}(\mathfrak{a})=\mathfrak{b}$. Now by part (i) of this theorem

$$
\mathfrak{a} \in \mathscr{S}_{h}\left(R_{\Delta}\right) \cap \times^{t} \Delta^{\infty}=\left(\mathscr{S}_{h} R\right)_{\Delta^{\infty}} \subseteq \bigcup\left\{\left(\mathscr{S}_{h} R_{i}\right)_{\Delta^{\infty}}: i<\omega\right\}
$$

so for some $j<\omega, \mathfrak{a} \in\left(\mathscr{S}_{h} R_{j}\right)_{\Delta^{\infty}} \subseteq \mathscr{S}_{h}\left(R_{j, \Delta}\right)$. But in turn this implies that $\mathfrak{b}=h_{k}^{*}(\mathfrak{a})$ $\in R_{j, \Delta}$ which proves our result (ii). Q.E.D.

$\mathscr{S S}_{0}^{0}(\Delta)$-THEOREM 15. Let $R, R_{i} \subseteq \times^{k} \omega$ for $i<\omega$ such that $R \subseteq \bigcup\left\{R_{i}: i<\omega\right\}$. (i) If each $R-R_{j}$ is unlimited then $\left|R_{\Delta^{\infty}}-\bigcup\left\{R_{i, \Delta^{\infty}}: i<\omega\right\}\right| \geqq c$ and (ii) if for some $I \subseteq k$ and $h: I \rightarrow \omega$ each $\mathscr{S}_{h}\left(R-R_{j}\right)$ is unlimited then $\left|R_{\Delta}-\bigcup\left\{R_{i, \Delta}: i<\omega\right\}\right| \geqq \mathrm{c}$.

Proof. Suppose each $R-R_{j}$ is unlimited. Let $g: \times^{2} \omega \sim \omega$ and let $A^{\#}$ be the real numbers conceived of as infinite sequences of 0 's and $i$ 's. If $\alpha, \beta \in A^{\#}$ let $\mu(\alpha, \beta)$ be the least integer $n$ such that $\alpha(n) \neq \beta(n)$ if $\alpha \neq \beta$, and equal to $\omega$ otherwise. Now for each $\alpha \in A^{\#}$ we construct $k$ unary nonconstant combinatorial functions $f_{j}^{\alpha}$ such that for $a \in \omega$ with $a=g(i, n), f^{\alpha}(a)=\left\langle f_{0}^{\alpha}(a), \ldots, f_{k-1}^{\alpha}(a)\right\rangle \in R-R_{i}$. Moreover we do this in such a way that if $\beta \in A^{\#}$ and $\mu(\alpha, \beta)<a$ we have $f_{j}^{\alpha}(a) \neq f_{j}^{\beta}(a)$ for each $j<k$. This can be done because the relation $R-R_{i}$ is unlimited and because as $\alpha$ ranges over $A^{\#}$ we need specify only $2^{a}$ different values $f^{\alpha}(a)$. Thus as $a$ ranges over $\omega$, $f^{\alpha}(a)$ remains in $R$ but avoids any given $R_{i}$ infinitely often. Moreover if $\alpha, \beta \in A^{\#}$ with $\alpha \neq \beta$ then $f_{j}^{\alpha}(a) \neq f_{j}^{\beta}(a)$ for all but finitely many $a \in \omega$. By Theorem 2 and Lemma $7 f_{\Delta}^{\alpha}(\mathfrak{a})=\left\langle f_{0, \Delta}^{\alpha}(\mathfrak{a}), \ldots, f_{k-1, \Delta}^{\alpha}(\mathfrak{a})\right\rangle \in R_{\Delta^{\infty}}$ for every $\mathfrak{a} \in \Delta^{\infty}$, and hence in particular for $\mathfrak{a}=\mathfrak{f}$, a universal cardinal. But $f_{\Delta}^{\alpha}(\mathfrak{t}) \notin R_{i, \Delta}$, for otherwise $f^{\alpha}(a) \in R_{\mathfrak{i}}$ for all but finitely many $a \in \omega$ which contradicts our construction. Moreover if $\alpha \neq \beta$ then $f_{\Delta}^{\alpha}(\mathfrak{t}) \neq f_{\Delta}^{\beta}(\mathfrak{t})$, for otherwise $f^{\alpha}(a)=f^{\beta}(a)$ for all but finitely many $a \in \omega$ which also contradicts our construction. Hence

$$
\left\{f_{\Delta}^{\alpha}(\mathfrak{t}): \alpha \in A^{\#}\right\} \subseteq R_{\Delta^{\infty}}-\bigcup\left\{R_{i, \Delta^{\infty}}: i<\omega\right\}
$$

has cardinality c. This proves (i). For (ii) suppose $I$ and $h$ are given such that $\mathscr{S}_{h}\left(R-R_{j}\right)$ is unlimited for $j<\omega$. By part (i) of this theorem there are at least $\mathrm{c}$ elements in $\left(\mathscr{S}_{h} R\right)_{\Delta^{\infty}}-\bigcup\left\{\left(\mathscr{S}_{h} R_{i}\right)_{\Delta^{\infty}}: i<\omega\right\}$, and by Lemma 6 for each such element $\mathfrak{a}, h_{k}^{*}(\mathfrak{a}) \in R_{\Delta}-\bigcup\left\{R_{i, \Delta}: i<\omega\right\}$. Our result (ii) then follows from the fact that $h_{k}^{*}$ is one one. Q.E.D.

Finally note the fact that if $R \subseteq \omega$ is the range of a unary nonconstant combinatorial function $f$, and $\mathfrak{t}$ is a universal cardinal then $f_{\Delta}(\mathfrak{t}) \in R_{\Delta}-\bigcup\left\{S_{\Delta}: R-S\right.$ infinite\}.

6. Horn sentences. By using the basic idea of [13] we can extend every relation $R \subseteq \times^{k} \omega^{*}$ to a relation $R_{\Delta^{*}} \subseteq \times^{k} \Delta^{*}$ by defining

$$
R_{\Delta} \cdot(\mathfrak{a}-* \mathfrak{b}) \text { iff } \tilde{R}_{\Delta}\left(\mathfrak{a}_{0}, \mathfrak{b}_{0}, \ldots, \mathfrak{a}_{k-1}, \mathfrak{b}_{k-1}\right) \text { for } \mathfrak{a}, \mathfrak{b} \in \times^{k} \Delta
$$

where $\tilde{R} \subseteq \times^{2 k} \omega$ is extended as in Definition 1 , and is defined by

$$
R\left(a-{ }^{*} b\right) \text { iff } \tilde{R}\left(a_{0}, b_{0}, \ldots, a_{k-1}, b_{k-1}\right) \text { for } a, b \in \times^{k} \omega .
$$

It is necessary to supply a lemma showing that $R_{\Delta}$. is well defined, but this presents 
no difficulty since the required property can be expressed by a Horn sentence and hence extends from $\omega$ to $\Delta$ by Theorem 2. In [6] we extended $R \subseteq X^{k} \omega^{*}$ to an $R^{\Delta^{*}} \subseteq \times^{k} \Delta^{*}$ by defining

$$
R^{\Delta^{\bullet}}=\left\{\mathfrak{a} \in X^{k} \Delta^{*}: f_{\Delta^{\bullet}}(\mathfrak{a})=0\right\}
$$

where $f$ is the characteristic function of $R$ with respect to $\times^{k} \omega^{*}$. In view of (14) and Theorem 1 we have $R_{\Delta^{*}}=R^{\Delta^{*}}$, and by Lemma 4

$$
\text { if } R \subseteq \times^{k} \omega \text { then } R_{\Delta^{*}} \cap \times^{k} \Delta=R_{\Delta} .
$$

If $\mathfrak{A}$ is a sentence of $L R$ and $\Sigma \subseteq \Delta^{*}$ let $\mathfrak{A}_{\Sigma}$ be the interpretation obtained by allowing variables to range over $\Sigma$, by letting functors denote extensions to $\Sigma$ of fixed functions over $\omega^{*}$, and by letting predicates denote extensions to $\Sigma$ of fixed relations over $\omega^{*}$. Then in [6] we proved that if $\mathfrak{A}$ is an arbitrarily quantified Horn sentence then

$$
\text { if } \mathfrak{A}_{\omega^{*}} \text { then } \mathfrak{A}_{\Delta^{*}}
$$

In [6] we extended every function $r: A \rightarrow B$ where $A \subseteq \times^{k} \omega^{*}$ and $B \subseteq \omega^{*}$ to a unction $r_{\Delta^{*}}: A_{\Delta^{*}} \rightarrow B_{\Delta^{*}}$ by defining

$$
r_{\Delta^{*}}(\mathfrak{a})=\mathfrak{b} \quad \text { iff } R_{\Delta^{*}}(\mathfrak{a}, \mathfrak{b}) \quad \text { for } \mathfrak{a} \in X^{k} \Delta^{*}, \mathfrak{b} \in \Delta^{*}
$$

where $R \subseteq X^{k+1} \omega^{*}$ is extended by (29) and is defined by

$$
r(a)=b \quad \text { iff } R(a, b) \text { for } a \in \times^{k} \omega^{*}, b \in \omega^{*} .
$$

Several lemmas are necessary in order to justify this definition: however, as the desired properties can be expressed by Horn sentences no difficulties arise. Let $\Delta^{+}=\omega_{\Delta^{*}}$. By (32) $\Delta \subseteq \Delta^{+} \subseteq \Delta^{*}$, moreover we can easily show that $\left|\Delta^{*}-\Delta\right|=\delta$, and in $\mathscr{S}_{1}^{0}(\Delta)$ that $\left|\Delta^{+}-\Delta\right| \geqq c$. Using Horn sentences and (33) we can also show that if $R \subseteq \times^{k} \omega$ then $R_{\Delta^{*}} \subseteq \times^{k} \Delta^{+}$and if $f: X^{k} \omega \rightarrow \omega$ then $f_{\Delta^{*}}: \times^{k} \Delta^{+} \rightarrow \Delta^{+}$. Thus $R_{\Delta^{+}}=R_{\Delta^{*}}$ and $f_{\Delta^{+}}=f_{\Delta^{*}}$. If $\mathfrak{Q}$ is a sentence of LR and $\Sigma \subseteq \Delta^{+}$let $\mathfrak{U}_{\Sigma}$ be the interpretation obtained by allowing variables to range over $\Sigma$, by letting functors denote extensions to $\Sigma$ of fixed functions over $\omega$, and by letting predicates denote extensions to $\Sigma$ of fixed relations over $\omega$. If $\mathfrak{A}$ is an arbitrarily quantified Horn sentence then we can easily show from (33) and the preceding remarks

$$
\text { if } \mathfrak{A}_{\omega} \text { then } \mathfrak{A}_{\Delta}{ }^{+} \text {. }
$$

We finally note that if $f: X^{k} \omega \rightarrow \omega$ and $a \in X^{k} \Delta$ then by (33) and Lemma 4 $f_{\Delta}(\mathfrak{a})=f_{\Delta^{+}}(\mathfrak{a})$.

A natural question to ask about a combinatorial $f: \omega \rightarrow \omega$ is "what are the conditions under which $\rho f_{\Delta}=(\rho f)_{\Delta}$ ?". A complete treatment of this question for recursive combinatorial $f$ with respect to the isols was obtained in [14]. We shall use those methods in order to obtain an answer to the corresponding problem in $\Delta$. A function $f: \omega \rightarrow \omega$ is called linear if $f(a)=r a+s$ for $a \in \omega$ where $r$ and $s$ are fixed 
elements of $\omega$. Eventually linear will have its usual meaning. We start out with the following

LeMma 8. Let $0<n<\omega$ and $\mathfrak{a} \in \Delta^{*}$. If $n \mathfrak{a} \in \Delta$ then $\mathfrak{a} \in \Delta$.

Proof. Let $\mathfrak{a}=\mathfrak{a}_{0}-\mathfrak{a}_{1}$ where $\mathfrak{a}_{0}, \mathfrak{a}_{1} \in \Delta$. Then by hypothesis there is a $\mathfrak{b} \in \Delta$ such that $n \mathfrak{a}=n \mathfrak{a}_{0}-n \mathfrak{a}_{1}=\mathfrak{b} \in \Delta$. Hence $n \mathfrak{a}_{1} \leqq n \mathfrak{a}_{0}$ so that by the cancellation law of [15] $\mathfrak{a}_{1} \leqq \mathfrak{a}_{0}$ giving $\mathfrak{a}=\mathfrak{a}_{0}-\mathfrak{a}_{1} \in \Delta$. Q.E.D.

THEOREM 16. Let $f: \omega \rightarrow \omega$ be eventually combinatorial. (i) $\rho\left(f_{\Delta}\right) \subseteq(\rho f)_{\Delta}$, and (ii) if $f$ is eventually linear then $\rho\left(f_{\Delta}\right)=(\rho f)_{\Delta}$.

Proof. If $R=\rho f$ then $(\forall a \in \Sigma) R_{\Sigma}\left(f_{\Sigma}(\mathfrak{a})\right)$ holds for $\Sigma=\omega$ and hence by Theorem 5 for $\Sigma=\Delta$. Thus $\rho\left(f_{\Delta}\right) \subseteq(\rho f)_{\Delta}$ proving (i). For (ii) suppose that for some $m, r, s \in \omega$, $f^{(m)}(a)=r a+s$ for $a \in \omega$. If $r=0$ then $\rho f$ is finite so by Corollary 2(iv) ( $\left.\rho f\right)_{\Delta}=\rho f$ $\subseteq \rho\left(f_{\Delta}\right)$. If $r \neq 0$ then there is a sufficiently large $n \in \omega$ such that if $R=\rho f$ then $(\forall \mathfrak{b} \in \Sigma)(\exists \mathfrak{a} \in \Sigma)\left(R_{\Sigma}(\mathfrak{b}+n) \rightarrow \mathfrak{b}+n=r \mathfrak{a}+s\right)$ holds for $\Sigma=\omega$ and hence by (36) for $\Sigma=\Delta^{+}$. Now consider any $\mathfrak{b} \in R_{\Delta}$. If $\mathfrak{b} \in \omega$ then by (5) $\mathfrak{b} \in R=\rho f \subseteq \rho\left(f_{\Delta}\right)$. If $\mathfrak{b} \notin \omega$ then $\mathfrak{b}=\mathfrak{b}^{\prime}+n$ for some $\mathfrak{b}^{\prime} \in \Delta$. Then $\mathfrak{b}^{\prime}+n \in R_{\Delta} \subseteq R_{\Delta^{+}}$so that $\mathfrak{b}=r \mathfrak{a}+s$ for some $\mathfrak{a} \in \Delta^{+}$. Now $r \mathfrak{a}=\mathfrak{b}-s \in \Delta$ and hence by Lemma $8 \mathfrak{a} \in \Delta$. Thus $\mathfrak{b}=f_{\Delta}^{(m)}(\mathfrak{a})=f_{\Delta}(\mathfrak{a}+m)$ $\in \rho\left(f_{\Delta}\right)$. But this proves our theorem. Q.E.D.

The principal result of [14] is that

there exists a strictly increasing $g: \omega \rightarrow \omega$ which is not eventually combinatorial such that for every eventually combinatorial $f: \omega \rightarrow \omega$ which is not eventually linear the composition $f \circ g$ is eventually combinatorial.

(S) $1(\Delta)$-THEOREM 17. If $f: \omega \rightarrow \omega$ is eventually combinatorial but not eventually linear then $(\rho f)_{\Delta}-\rho\left(f_{\Delta}\right)$ is nonempty.

Proof. Since $f$ is eventually combinatorial but not eventually linear there is an $m \in \omega$ such that $f^{(m)}$ is a strictly increasing combinatorial function and $f(m)>f(a)$ for every $a<m$. Let $g$ be the function of (37) where without loss of generality we assume that $g(0)>m$. If $R=\rho f$ and $h=f \circ g$ then $h$ is eventually combinatorial so that $(\forall \mathfrak{a} \in \Sigma) R_{\Sigma}\left(h_{\Sigma}(\mathfrak{a})\right)$ holds for $\Sigma=\omega$ and hence by Theorem 5 for $\Sigma=\Delta$. If $\mathfrak{t}$ is strongly universal then $h_{\Delta}(\mathfrak{f}) \in R_{\Delta}$. Since $g$ maps into $\omega$ and $\mathfrak{t}$ is strongly universal $g_{\Delta}(\mathfrak{l}) \in \Delta^{+}-\Delta$ and consequently $h_{\Delta}(\mathfrak{f})=f_{\Delta^{+}}\left(g_{\Delta}(\mathfrak{f})\right)$. Finally we must show that $h_{\Delta}(\mathfrak{f})$ is not in the range of $f_{\Delta} .(\forall \mathfrak{a}, \mathfrak{b} \in \Sigma)\left(f_{\Sigma}\left(g_{\Sigma}(\mathfrak{a})\right)=f_{\Sigma}(\mathfrak{b}) \rightarrow g_{\Sigma}(\mathfrak{a})=\mathfrak{b}\right)$ holds for $\Sigma=\omega$ and hence by (36) for $\Sigma=\Delta^{+}$. If $h_{\Delta}(\mathfrak{l})=f_{\Delta}(\mathfrak{b})$ for some $\mathfrak{b} \in \Delta$ then $f_{\Delta^{+}}\left(g_{\Delta^{+}}(\mathfrak{t})\right)$ $=f_{\Delta}+\left(g_{\Delta}(\mathfrak{t})\right)=f_{\Delta}(\mathfrak{b})=f_{\Delta^{+}}(\mathfrak{b})$ so that $\mathfrak{b}=g_{\Delta^{+}}(\mathfrak{l})=g_{\Delta}(\mathfrak{l}) \notin \Delta$ which is a contradiction. Q.E.D.

We have included full proofs of the last two theorems, despite their similarity to the isolic proofs of [14], because of the detailed information they shed on transfer theorems for Horn sentences. Let us first recall that by Theorem 5 universally quantified Horn sentences extend from $\omega$ to $\Delta$. That that is not the case for 
arbitrarily quantified Horn sentences is a simple consequence of Theorem 17. For let $S=\left\{a^{2}: a \in \omega\right\}$.

$$
(\forall \mathfrak{a} \in \Sigma)(\exists \mathfrak{b} \in \Sigma)\left(S_{\Sigma}(\mathfrak{a}) \rightarrow \mathfrak{a}=\mathfrak{b}^{2}\right)
$$

holds for $\Sigma=\omega$, is false for $\Sigma=\Delta$ in $\mathscr{S S}_{1}^{0}(\Delta)$, and consequently is not a theorem for $\Sigma=\Delta$ in $\mathscr{S}^{\circ}$ (provided $\mathscr{S}$ is consistent). In general the only way (with the single exception of (42) below) of showing that an arbitarily quantified Horn sentence extends from $\omega$ to $\Delta$ is to show that it holds in $\omega$ with almost combinatorial Skolem functions, and then use Theorem 5 to extend the resulting universal Horn sentence to $\Delta$. Thus

$$
(\forall \mathfrak{a} \in \Sigma)(\exists \mathfrak{b} \in \Sigma)(\mathfrak{a}(\mathfrak{a}+1)=2 \mathfrak{b})
$$

holds for $\Sigma=\omega$, and is true for $\Sigma=\Delta$ in $\mathfrak{B S}^{0}$. This follows because $f(a)=\left(\begin{array}{c}a+1 \\ 2\end{array}\right)$ is combinatorial and $(\forall \mathfrak{a} \in \Sigma)(\mathfrak{a}(\mathfrak{a}+1)=2 f(\mathfrak{a}))$ holds for $\Sigma=\omega$, hence by Theorem 5 for $\Sigma=\Delta$, and thus implies (39) for $\Sigma=\Delta$. A natural conjecture might be that this is the only way of getting a transfer theorem for quantified sentences. And indeed we have partial results in this direction. Let $\varsigma^{0}$ be a theory just like $\mathbb{S}^{0}$ except that urelemente are admitted and let $\mathfrak{A}$ be an arbitrarily quantified sentence whose matrix is a single equation between polynomials built up from variables and functors for + and $\cdot$. Then in [8] we found that $\mathfrak{A}_{\Delta}$ is a theorem of $\mathfrak{S}^{0}$ if and only if we can prove in $\mathfrak{S}$ that $\mathfrak{A}_{\omega}$ holds with almost combinatorial Skolem functions. It is a sad consequence of Theorem 16 that no such theorem could hold for arbitrarily quantified Horn sentences. For if we let $E=\{2 a: a \in \omega\}$ then

$$
(\forall \mathfrak{a} \in \Sigma)(\exists \mathfrak{b} \in \Sigma)\left(E_{\Sigma}(\mathfrak{a}) \rightarrow \mathfrak{a}=2 \mathfrak{b}\right)
$$

holds for $\Sigma=\omega$, and is true for $\Sigma=\Delta$ in $\mathbb{B}^{\circ}$. If we examine the Skolem function $f$ for (40) we see that it cannot be almost combinatorial. For otherwise there would be an $m \in \omega$ such that $a \leqq f^{(m)}(a)=(a+m) / 2$ for all $a \in \omega$ with $a+m \in E$. Thus (40) extends from $\omega$ to $\Delta$ without an almost combinatorial Skolem function. Perhaps this has something to do with the linearity in the consequent of (40). This too is sadly not the case, for in [5], using only the notion of a universal cardinal, we found

$$
(\forall \mathfrak{a}, \mathfrak{b}, \mathfrak{c} \in \Sigma)(\exists \mathfrak{d} \in \Sigma)\left(\mathfrak{a}^{2}=\mathfrak{b}^{2}+\mathfrak{c} \rightarrow \mathfrak{a}=\mathfrak{b}+\mathfrak{d}\right)
$$

holds for $\Sigma=\omega$, is false for $\Sigma=\Delta$ in $\left(S_{0}^{0}(\Delta)\right.$, and hence is not a theorem for $\Sigma=\Delta$ in $B^{\circ}$. Thus the only remaining place where we might reasonably find a transfer theorem from $\omega$ to $\Delta$ without almost combinatorial Skolem functions is when both antecedent and consequent are linear. We can finally and happily say "that is the case", for in [1] it is shown that for any sentence $\mathbb{C}$ having the form

$$
\left(\forall \mathfrak{a} \in X^{k} \Sigma\right)\left(\exists \mathfrak{b} \in X^{n} \Sigma\right)(\mathfrak{H}(\mathfrak{a}) \rightarrow \mathfrak{B}(\mathfrak{a}, \mathfrak{b}))
$$

where $\mathfrak{A}$ and $\mathfrak{B}$ are conjunctions of linear homogeneous equations in the indicated variables, we can prove in $\mathfrak{S}$ that $\mathfrak{A}_{\omega}$ implies $\mathfrak{A}_{\Delta}$. We thus see the remarkable 
differences between the linear and nonlinear theories of $\Delta$ exemplified by (38) and (40).

\section{REFERENCES}

1. R. Bradford, Undecidability of the theory of Dedekind cardinal addition, Summer Institute on Axiomatic Set Theory, (Los Angeles, 1967) Amer. Math. Soc., Providence, R. I.

2. P J. Cohen, Set theory and the continuum hypothesis, Benjamin, New York, 1966. MR 38 \#999.

3. J. C. E. Dekker, A non-constructive extension of the number system, J. Symbolic Logic 20 (1955). 204-205.

4. E. Ellentuck, The theory of Dedekind finite cardinals, Dissertation, Univ. of California, Berkeley, Calif., 1962.

5. - The universal properties of Dedekind finite cardinals, Ann. of Math. (2) 82 (1965), 225-248. MR 31 \#4729.

6. — , The first order properties of Dedekind finite integers, Fund. Math. 63 (1968), $7-25$.

7. - A choice free theory of Dedekind cardinals, J. Symbolic Logic 33 (1968), 60-84.

8. - Almost combinatorial functions, Notices Amer. Math. Soc. 15 (1968), 650-651. Abstract \#68T-500.

9. K. Gödel, The consistency of the continuum hypothesis, Ann. of Math. Studies, no. 3, Princeton Univ. Press, Princeton, N. J., 1940. MR 2, 66.

10. A. Lévy, Independence results in set theory by Cohen's method. I-IV, Notices Amer. Math. Soc. 10 (1963), 592-593. Abstract \#63T-388.

11. J. Myhill, Recursive equivalence types and combinatorial functions, Bull. Amer. Math. Soc. 64 (1958), 373-376. MR 21 \#7.

12. A. Nerode, Extensions to isols, Ann. of Math. (2) 73 (1961), 362-403. MR 24 \#A1215.

13. - - Extensions to isolic integers, Ann. of Math. (2) 75 (1962), 419-448. MR 25 \#3830.

14. - Non-linear combinatorial functions of isols, Math. Z. 86 (1965), 410-424. MR 34 \#5672.

15. A. Tarski, Cancellation laws in the arithmetic of cardinals, Fund. Math. 36 (1949), 77-92. MR 11, 335.

Rutgers, The State University,

New BRUNSWICK, New JeRSEy 08903

KYOTO UNIVERSITY,

KYOTO, JAPAN 